How to reference this article Mariani, L. (2019). Dopo l'Ottocento delle attrici, qualche punto fermo sulle attrici italiane del Novecento. Italica Wratislaviensia, 10(2), 195-222.

DOI: http://dx.doi.org/10.15804/IW.2019.10.1.25

Saggio introduttivo

\author{
Laura Mariani \\ Università degli Studi di Bologna \\ annalaura.mariani@unibo.it \\ ORCID: 0000-0002-4305-9884
}

\title{
DOPO L'OTTOCENTO DELLE ATTRICI, QUALCHE PUNTO FERMO SULLE ATTRICI ITALIANE DEL NOVECENTO
}

\author{
AFTER THE 1800's, “CENTURY OF ACTRESSES", \\ A FEW CORE POINTS ON ITALIAN ACTRESSES \\ IN THE 1900's
}

\begin{abstract}
Critical studies on the theatre from the $19^{\text {th }}$ and $20^{\text {th }}$ centuries, even founding ones, are limited as they do not include a gender category - a paradox in a space where bodies are central. The actresses, in fact, give life to different stories, which allow for the enrichment of historical-critical reconstructions. Given the vastness of the topic, this article aims to identify some fundamental turning points. We begin with the legacy of the $19^{\text {th }}$ century, rich from both a linguistic and a historical-critical viewpoint; the article moves on to the post-Duse period, which saw, on the one hand, the birth of the actor-artist's Italian specificity and, on the other, the crisis of female leaders of theatre companies; then, the article discusses the birth of Italian theatre direction in the period following the Second World War and the reaction of a few actresses, like Anna Magnani, who aimed to re-create the reality that was to be reclaimed by the history of theatre. Finally, the article explores the breakdown caused by the New Theatre and even calls into discussion the word for "actress", with overlaps in the fields of theatre direction and dramaturgy.
\end{abstract}

Keywords: $19^{\text {th }}$ century, $20^{\text {th }}$ century, theatre, actress, history of women 


\section{PREMESSA}

$\mathrm{U}$ na visione d'insieme dell'Ottocento teatrale dal punto di vista della Storia delle donne è possibile. Le attrici di due secoli fa hanno lasciato un'eredità importante, a mio avviso declinabile lungo due direzioni: una riguarda l'elaborazione di elementi recitativi precorritori, connotati dal genere, e l'altra la capacità consolidata di occupare lo spazio pubblico tradizionalmente maschile, fino all'esercizio anomalo di potere attraverso il capocomicato. Uno sguardo sintetico di questo tipo è possibile per il Novecento?

La difficoltà maggiore dipende dalle caratteristiche generali del secolo, di rara intensità: aperto dalla prima guerra mondiale della storia e concluso da una rivoluzione globale, che mette alla prova la capacità del teatro, questa antica struttura di civiltà, di essere arte del nostro presente social. In mezzo una dittatura, la seconda guerra mondiale e la lotta partigiana, la ricostruzione e il boom economico, la nascita dell'Unione europea e la globalizzazione, la conquista dello spazio e gli straordinari progressi della scienza, le migrazioni di massa e il peggioramento dello stato di salute del pianeta... Tragedie e conquiste si mescolano incessantemente, ne consegue che non sappiamo «cosa lo definisce. Sappiamo che il Novecento è il secolo di tutte le opposizioni, di ogni ordine e di ogni disordine; ma non ne conosciamo lo stile, il comportamento» (Garboli, 1998, p. 303). Un aspetto che si conferma assumendo il punto di vista femminile: «il secolo più sanguinoso della storia dell'umanità e quello in cui le donne occidentali, molto dopo gli uomini, accedono alla modernità», una modernità definita da alcune conquiste oggettive e ancor più dalla «conquista di una posizione da soggetto, da individuo a pieno diritto, da cittadina» e da nuovi rapporti fra i sessi, dove però le disparità si riproducono, persistono, cambiano aspetto, si diversificano in un orizzonte che va oltre le donne bianche dell'occidente (Thebaud, 1992, pp. 3-17).

Una difficoltà specifica riguarda il fatto che gli studi teatrali hanno comprensibilmente privilegiato gli aspetti di rottura prodotti dalla rivoluzione registica e dalle avanguardie, all'inizio e poi nella seconda metà del secolo, fenomeni in cui la presenza femminile non è imme- 
diatamente evidente. E dunque occorre formulare nuove domande, perché si produce quel fenomeno di assenza/presenza analizzato da Marina Zancan per la storia letteraria: dove da un lato le numerose donne che hanno scritto rimangono escluse dal sistema letterario, dalle sue regole e dalle sue scale di valori, e, dall'altro, è innegabile la presenza di una soggettività femminile attiva nell'esercizio della scrittura. Un approccio prezioso se si guarda alla drammaturgia e alla regia.

Anche a un primo sguardo, invece, le attrici continuano a esserci in gran numero e da protagoniste, ma non è facile farne storia dopo Eleonora Duse. Per l'Ottocento possediamo una catena di maestre indiscusse, per il Novecento su chi dobbiamo puntare per capire come cambiano i linguaggi, i processi, le condizioni di lavoro? Non sono molte le ricostruzioni dei percorsi biografici e artistici individuali, indispensabili per riempire di sostanza i nomi che ci sono restati, sia per il teatro di prosa che per i generi cosiddetti minori, senza considerare la danza e l'opera. Persino il ricco panorama degli studi critici, da questo punto di vista, è sconfortante: la categoria di gender viene pressoché ignorata, ed è paradossale in un ambito in cui il corpo è centrale.

Anche per questo è importante individuare alcuni nodi problematici (e alcune figure) che ci orientino nell'attraversamento. Sono di per sé evidenti alcune fasi storiche e teatrali: il dopo Duse, la fine della cosiddetta microsocietà degli attori, la mutata condizione delle attrici; il secondo dopoguerra e le attrici che agiscono (e reagiscono) nel teatro di regia, in concomitanza con i successi del cinema; le protagoniste del Nuovo teatro, in prossimità e dopo il ' 68 , e la messa in discussione delle identità tradizionali. Sarà una ricostruzione a volo d'uccello, costretta fra ampiezza dei temi e ristrettezza degli spazi: limiti accresciuti dalla vicinanza per il terzo snodo, che si chiude alla fine degli anni Ottanta, seguendo l'idea di secolo "breve" di Hobsbawm.

\section{IL RICCO LASCITO OTTOCENTESCO}

Del Sette-Ottocento teatrale, solitamente visto come passaggio fra le due epoche d'oro della Commedia dell'Arte e della Regia, Claudio Meldolesi ha felicemente ricostruito l'identità autonoma dandole un nome, 
«età delle invenzioni $»^{1}$. Ora però, concentrandoci sul secolo del Grande attore, intendiamo sottolineare che non se ne vede tutta la capacità inventiva se non si considera il ruolo svolto dalle donne, nella doppia veste di attrici e di spettatrici. Me ne sono occupata in un saggio di cui riprendo alcune acquisizioni di fondo (Mariani, 2017).

Partiamo dalle protagoniste, considerando che hanno attorno a sé attrici notevoli con percorsi meno originali, studiate poco o niente: infatti, da un insieme di tale tipo, che dalle eccellenze arriva alla medietà, si definisce una civiltà teatrale. La catena è inaugurata da Carlotta Marchionni (1796-1861), l'attrice del primo Romanticismo che fonda «il paradigma dell'attrice moderna» (Geraci, 2010): inaugura il teatro del personaggio concepito come «identità psichica unitaria», realizzata scenicamente «secondo criteri di verisimiglianza e storicità», e costruisce un'immagine pubblica che rovescia gli stereotipi, figlia d'arte che s'impone come «attrice vergine e santa» (Aliverti, 1992, pp. 22 e 202-203). Adelaide Ristori (1822-1906), pure figlia d'arte, divenuta col matrimonio marchesa Capranica del Grillo, appartenente alla triade del Grande attore, conquistatrice dei mercati internazionali con le sue "donne-mondo" e incarnazione di fatto del diritto femminile alla cittadinanza, è protagonista di punta di una possibile storia delle attrici ${ }^{2}$. Giacinta Pezzana (1841-1919), esponente del tardo romanticismo e del naturalismo con venature espressioniste, viene considerata in questa catena soprattutto come attrice dell'emancipazionismo per il suo impegno militante e per come ha gestito la sua carriera e la sua stessa vita: mettendo la libertà prima del successo, l'etica prima del guadagno, le relazioni profonde prima di quelle utili (Mariani, 2005). Infine, Eleonora

1 «Invenzione del teatro come edificio riproducibile, invenzione della 'moderna' teoria dell'attore, invenzione della 'nuova' sensibilità recitativa che collegò Lumi e Romanticismo oltre la logica dei generi, invenzione di rapporti riformati fra attore e autore, invenzione di modalità partecipative aperte, invenzione di modalità rappresentative alte e basse allo stesso tempo, invenzione di una nuova competitività borghese fra prosa e lirica, arte varia e circhi» (Meldolesi, 1995, pp. 413-414).

${ }^{2}$ La studiosa maggiore di Ristori è Teresa Viziano, che ha curato il suo ricco archivio custodito presso il Museo Biblioteca dell'Attore di Genova, oltre a scrivere numerosi saggi. 
Duse (1858-1924), che rappresenta sia l'apice della cultura tradizionale dell'attore italiano sia una figura nuova di attrice-artista: mossa da istanze utopiche al pari dei padri fondatori della Regia e in cerca di una verità scenica che sappia cogliere i livelli frammentari, contraddittori, nascosti del Novecento come «secolo del Grottesco»; per suo tramite Mejerchol'd comprende «che la recitazione è un'arte femminile, o meglio del femminile» (Attisani, 2017, pp. 128-160 e Idem, 2015, p. 315)훙 su di lei si è scritto a livello internazionale e si continua a scrivere.

Partiamo dalla dicotomia segnalata da Meldolesi fra il Grottesco di Gustavo Modena e il Romanticismo di Adelaide Ristori, fra il primattore che tende a diventare caratterista e la primattrice che esprime l'anima negativa del Romanticismo (Meldolesi, Taviani, pp. 244-258): due linguaggi, due tendenze forse non casualmente attribuibili ad artisti di sesso diverso, forse non spiegabili solo attraverso il legame convenzionalmente stabilito fra femminile e mondo dei sentimenti di contro al maschile/razionale. In questo senso, assume peso una condizione particolare vissuta dalle attrici: la necessità di fornire di sé un'immagine socialmente positiva, almeno in apparenza regolare, tale comunque da non essere compromessa dall'interpretazione di personaggi poco rassicuranti. L'attore non era obbligato a giustificare il fatto di interpretare Macbeth, l'attrice doveva dar conto della scelta di incarnare la Lady, non solo a parole e con comportamenti privati agli antipodi, ma in scena, a partire dal personaggio stesso, dissociandolo da sé. Questa sorta di allenamento alla complessità favoriva la ricerca di dettagli utili e l'immissione nella recitazione di elementi di sottorecitazione e di antirecitazione, sia attraverso la mimica sia con la creazione di frammenti indimenticabili o in opposizione: si pensi all'immagine di madre amorosa, protettiva, perseguitata con cui Adelaide Ristori iniziava la sua Medea. Dunque, se il grottesco è la chiave della composizione dell'attore novecentesco, vi contribuisce una pratica alla quale l'attrice è spinta dal suo essere donna, con un salto nell'uso e nella consapevolezza compiuto da Eleonora Duse.

3 Lo studioso considera sia i processi compositivi messi in atto da Duse che la sua tensione ad andare oltre, trovare «la vita nella forma», spiazzare il pubblico. 
Si tratta per lo più di dettagli: i giochi di sguardi e i «petit riens» di Carlotta Marchionni; i rantoli di Adelaide Ristori che nella scena del sonnambulismo di Lady Macbeth russava; le parole ripetute in vari toni da Giacinta Pezzana con effetti wagneriani e i suoi «piccoli incisi» che sembravano «tormentosi commenti e inaspettate rivelazioni» (Zacconi, 1946, p. 61). Eleonora Duse nella Signora della camelie arrivava a invocare undici volte Armando ed era solita ricorrere a gesti incongrui, inaspettati, appresi osservando i comportamenti quotidiani delle donne ${ }^{4}$. Si tratta di tic, di inezie, come quelle che vediamo enfatizzate nei primi piani cinematografici di Meryl Streep.

I primi piani appunto, che soprattutto nei palcoscenici poco illuminati del passato sono frutto di abilità recitativa. Ferdinando Taviani parla del «corpo delle meraviglie» degli attori e del volto delle attrici come emisferi complementari: «perché è attraverso le attrici soprattutto che il volto umano diventa un paesaggio misterioso e dalle molteplici dimensioni», da cui affiorano i moti dell'anima. D'altro canto, come il volto può essere locus dell'accadere per l'attore, così l'attrice può stupire con un «corpo delle meraviglie»: il sorriso di Eleonora Duse «si irradiava per tutto il corpo» (Schino, 2008, p. 391), la sua recitazione sembrò acrobatica alla Ristori, del potere di seduzione connesso all'esposizione scenica di sé fece uno dei tratti sconvolgenti della sua recitazione. In continuazione toccava i suoi compagni in scena e la sua Margherita Gauthier baciava Armando sulla bocca, non sulla fronte.

Un eros particolare emanava poi dall'attrice en travesti, una pratica inaugurata dalle comiche dell'arte, rifiutata da Ristori e Duse e riavvalorata da Sarah Bernhardt. Nella contrainte di un tragitto volto a incarnare un'altra identità sessuale senza perdere la propria, l'attrice è portata infatti a lavorare in dettaglio e in profondità, per sottrazioni e «petit

${ }^{4}$ «Le famose interiezioni della Duse erano il cuore del suo modo di recitare. Erano, tra l'altro, il modo principale in cui un'attrice del suo calibro si permetteva di riplasmare un testo [...e] creava la vita dei suoi celebri silenzi. Erano l'equivalente di quei suoi continui gesti strani tante volte ricordati, $[. .$.$] come una persona in situazione privata,$ e così faceva nascere nello spettatore il dubbio persino angoscioso che non stesse contemplando un'attrice in scena, ma che stesse spiando un essere umano in difficoltà da un buco della serratura» (Schino, 2016, p. 203). 
riens». Anche l'attore che si traveste può attuare un processo analogo, che parte dalla ricerca del femminile dentro di sé, ma minori sono gli stimoli ad alleggerire perché la femminilità stessa è stata concepita e costruita come «una mascherata» (Rivière, 1991, pp. 90-101).

Sia che si tratti di donne in scena sia che si tratti di attori travestiti da donne, a provocare turbamento è il femminile, che sembra riguardare l'occhio del pubblico prima che il viso guardato. Qualcosa che non si basa tanto sulla bellezza fisica in sé quanto sulla presenza scenica e sulla trasfigurazione, il fascino del «je ne sais quoi» delle attrici italiane ${ }^{5}$. $\mathrm{Nel}$ patto continuamente rinnovato fra naturale e artificiale il femminile muove dai colori patetici di un Romanticismo che si prolunga oltre le sue fortune letterarie per assumere a fine secolo tratti esaltati, fra figure sublimate e nuovi "idoli di perversità", comprese le femministe.

D'altro canto, le attrici in carne ed ossa non solo fanno sognare le altre donne con i loro personaggi ma si pongono come modelli: lavorano, possono affermarsi, godono di libertà, occupano disinvoltamente la sfera pubblica, incarnano la Donna nuova. Non senza costi e lacerazioni, perché l'attrice è intimamente attraversata dal conflitto fra il suo mestiere e la "natura femminile", questo costrutto culturale così invasivo: fatica più dell'attore a conciliare sfera privata e sfera pubblica e ad essere riconosciuta come cittadina.

A dispetto dell'apparenza, che ha reso schiacciante il modello di perfezione femminile da lei incarnato, è Adelaide Ristori a creare una tensione positiva e proficua tra arte scenica e immagine pubblica: afferma l'artisticità della professione e la sua dimensione imprenditoriale, la dignità dell'attrice e la sua capacità di proporsi come modello, non prescinde mai dalla dimensione pubblica e rende visibili momenti della sua vita privata, come i sovrani, è portavoce di Cavour e dei Savoia all'estero e rappresenta la patria per gli emigrati ${ }^{6}$. Dalla sua battaglia

5 Un' «arte di piacere» fatta di spontaneità, gaiezza, follia e basata sullo «spostamento di un richiamo erotico», scrive Meldolesi, 2013, pp. 1-21.

6 Anche lo spostamento di interesse dalla vita privata delle attrici alla loro qualità artistica, segnalato da Ciotti Cavalletto, è testimonianza di un processo emancipativo in parte inevitabile. 
discendono la libertà della Pezzana di essere un'attrice ribelle e quella della Duse di essere un'artista tout court.

Ma la società stessa presenta fenomeni di «femminizzazione» ${ }^{7}$ con conseguenze sull'offerta drammaturgica e spettacolare, perché l'industrializzazione favorisce l'ingresso delle donne nel mondo del lavoro e una loro maggiore visibilità mentre si apre l'accesso a professioni non solo manuali e le spettatrici diventano più numerose. Anche se il pubblico teatrale ottocentesco è presentato come maschile e la formazione di quello femminile non viene tematizzata nemmeno da Habermas, non è di poco conto osservare il processo che porta alla normalità dei pubblici misti novecenteschi e alla loro rappresentazione, a partire dall'acquisita consapevolezza della spettatrice di non dover necessariamente solo accompagnare qualcuno o esserne accompagnata ${ }^{8}$.

Certo le attrici non hanno mai pensato al pubblico in modo indifferenziato, ma il salto di qualità avviene con Giacinta Pezzana, per il suo impegno politico e le sue relazioni con mazziniane, emancipazioniste e filantrope, e ancor più con Eleonora Duse, quando dal rapporto con singole spettatrici o con frange politicizzate si passa a una concezione larga del pubblico come entità riconoscibile nelle sue componenti sessuate: con un occhio di riguardo ai «gusti delle femminuccie» che «fanno sorridere li maschietti, Shasperiani» e certa «canaglia di maschi». L'attrice costruisce con «le donne finte» delle commedie un dialogo ravvicinato, che prepara e garantisce quello con le spettatrici e fa delle relazioni amicali una poetica e un modo di lavorare (Duse, Boito, 1979, pp. 123, 585).

7 Cfr. O. Weininger, Geshlecht und Character, Wien: Braumüller, 1903. Egli segnala anche i grandi «successi femministi» del Rinascimento e del XVI secolo, quando comparvero le prime attrici.

8 Lo stesso Habermas, nella prefazione alla nuova edizione della sua Storia e critica dell'opinione pubblica (Bari-Roma: Laterza, 2002) riconosce di aver concepito la sfera pubblica borghese al singolare e di non aver considerato le donne: «diversamente dall'esclusione degli uomini emarginati, l'esclusione delle donne ha esercitato una funzione strutturale», precisa. 


\section{IL DOPO-DUSE: LA SPECIFICITÀ ITALIANA DELL'ATTORE-ARTISTA E LA CRISI DEL CAPOCOMICATO FEMMINILE}

Eleonora Duse torna a recitare nel 1921, dopo sedici anni di lontananza dalle scene, e muore nel 1924 a Pittsburgh, durante una faticosa tournée. In Italia non trova una collocazione adeguata alle sue necessità artistiche, ai suoi bisogni di vita, al suo nome. Saldamente padrona del mestiere posseduto da figlia d'arte, è insofferente delle miserie dell'ambiente teatrale e della sua routine, nutre un bisogno di purezza che è in contraddizione con la natura stessa del teatro, inquietudini tutte che alimentano l'unicità della sua recitazione: «attrice artista», secondo una definizione di Mario Apollonio9 rilanciata da Claudio Meldolesi.

Lascia un'eredità difficile da gestire in un contesto sempre più complesso. Una crisi epocale sta causando il crollo di un assetto originatosi con la Commedia dell'arte ed evolutosi certo, ma senza compromettere le sue strutture portanti, basate sulla compagnia capocomicale e su un intreccio inusual arte-vita: famiglia d'arte e sistema dei ruoli, nomadismo e trasmissione orale del sapere, organizzazione interna in funzione della produzione di spettacoli ed elaborazione di una cultura che va oltre l'evenemenzialità e che tutela la differenza dei suoi membri, comprese le opportunità di autorealizzazione offerte alle donne, una sorta di microsocietà ${ }^{10}$. È in atto un processo di industrializzazione che provoca lo scontro dei soggetti coinvolti - capocomici, attori, autori, proprietari dei teatri, dei repertori, dei capitali - mentre il fascismo, consapevole dell'importanza del teatro per la formazione dell'opinione pubblica, mette sotto tutela il teatro e ne determina gli indirizzi, inaugurando l'epoca delle sovvenzioni.

Prime vittime gli attori: quando si spezza «il filo tra organizzazione, gestione degli attori e creazione artistica» e a capo della compagnia si

9 Duse tenta «una ricerca unitaria, che trasfiguri in presenza spirituale ed in poesia gli innumerevoli paragrafi delle esperienze tecniche, le innumerevoli seduzioni delle immaginarie creature». «La persona umana veniva ad acquistare un senso decisivo nell'arte teatrale» (Apollonio, 2003, pp. 663 e 662).

${ }^{10}$ Cfr. Taviani, Schino, 1982 e Meldolesi, 2013, pp. 57-77. 
colloca un impresario-finanziatore, il primattore diventa uno scritturato (Pedullà, 1994, p. 126.). L' «aspra guerra al capocomicato» apre le porte alla «guerra al mestiere»: «era una vera razza che stava per perdere la sua fisionomia antica e gloriosa e che doveva, o decidersi a prenderne una assolutamente diversa, o ritirarsi in buon ordine rassegnata e vinta!», scrive Virgilio Talli (1927, pp. 238-239, 231). La crisi esplode negli anni trenta, ma giustamente Mirella Schino ne anticipa le origini agli inizi del secolo in forza di alcuni aspetti da riconsiderare: la cultura dell'attore di tradizione fu denigrata e lentamente svuotata fino alla perdita della memoria stessa di ciò che era stato, la cultura comunitaria e indipendente della microsocietà fece spazio all'omologazione sociale e all'occultamento delle differenze ${ }^{11}$. L'avvento della regia trovò attori individualizzati e rassegnati.

Le attrici in particolare come vissero questa condizione? L'anomalia della condizione femminile nella microsocietà teatrale era in conflitto con l'esaltazione fascista della casalinga-moglie-madre, tanto che alcune ritennero il proprio lavoro inconciliabile con la maternità e tornarono nei ranghi rinunciando al capocomicato. Che donne diverse dalle altre come le attrici si esponessero per il divertimento collettivo era infatti possibile, addirittura gradito, ma era inaccettabile che comandassero veramente, come era stato per Adelaide Ristori (che si definiva un comandante d'armata mentre a Parigi il marchese Capranica del Grillo era chiamato monsieur Ristori) o Giacinta Pezzana (che preferì dirigere compagnie minori anziché guadagnare di più come scritturata in compagnie primarie) o Eleonora Duse (una donna sola al comando) ${ }^{12}$.

Paola Borboni (1900-1995) diventa capocomica nel 1934 e smette nel 1939, scontrandosi con una crisi «di teatro tremenda, una crisi di pubblico» (c'era stata la guerra d'Etiopia, si avvicinava il secondo conflitto mondiale, «cambiava il mondo») (Mariani, 1987) ${ }^{13}$. Torna ca-

11 Si veda il ricco dossier prodotto dal gruppo di ricerca coordinato da Schino, che è ripartito dalle fonti e ha guardato soprattutto agli attori (Schino, Di Tizio, Legge, Marenzi, Scappa, Gaborik, Taddeo, Ponzetti).

12 Per il primo caso cfr. Ristori, 1887, per il secondo Mariani, 2005 e per il terzo almeno Simoncini, 2011.

${ }^{13}$ Ho intervistato Paola Borboni il 20 ottobre 1984 a Roma. 
pocomica nel 1942 e poi nel 1946 per mettere in scena Pirandello, si rovina economicamente e si rassegna infine a fare prestazioni occasionali senza legarsi a una compagnia fissa, recitando con successo fino a tarda età. Quando le domando se dà un giudizio positivo di quell'esperienza risponde: «Di me, io lo do di me, positivo, brava Paola!». Alla domanda se creava problemi che a comandare fosse una donna replica: «Capocomico una donna! Aspettavano i soldi come se io fossi un uomo, e io glieli ho dati, ma tanto io ero abituata a mantenere una famiglia, era diventata una famiglia un po' più grande». E a proposito della Pirandelliana, dice di aver «ricevuto» nel suo «cuore» Pirandello a ventitré anni e di averlo potuto riprendere solo nella maturità: «io non ho subito il fatto fisico tremendo della menopausa, tanto io ho lavorato in quegli anni intorno a questo autore che mi aveva quasi mangiato il cervello» (Ibidem). Emergono elementi, che troviamo sempre o quasi nelle imprese femminili di maggior peso: la passione, il coinvolgimento totale fin del corpo, il senso che essendo donne bisogna essere ineccepibili, fino a dare tutto ciò che si possiede.

A durare di più è il capocomicato di Emma Gramatica (1874-1965), più di mezzo secolo a partire dal 1903. Non è qui il caso di trattare il "caso Cortese" - quel Luca Cortese che sembrava volesse rivoltare il teatro italiano e si rivelò un truffatore - ma va ricordato che Emma, in nome della sua dignità e dei suoi doveri nei confronti della compagnia, prese pubblicamente posizione, da «solitaria ribelle» che era stata messa «nell'assoluta impossibilità di far da sola»: «Oggi che ho dei dati e dei fatti che mi provano quello che in linea generale era sempre apparso e cioè il pericolo enorme di accatastare teatri ed aziende nelle mani di una sola società, oggi m'oppongo al trust». Ebbe il plauso pubblico di Ermete Zacconi, il più agguerrito difensore del teatro d'attore, per aver preso risolutamente posizione «contro l'affarismo funesto» e aver difeso il suo diritto e la sua libertà («Così tutti i capocomici seguissero il vostro esempio ed ascoltassero le mie parole!»), mentre l'arte drammatica moriva «di anemia per scarsità di cibo e di ideale», l'affarismo l'avrebbe ridotta «ad un numero di varietà per i suoi caffè concerto» e i teatri delle grandi tradizioni si sarebbero aperti soprattutto «a spettacoli popolari o a proiezioni cinematografiche» (Schino, 1997, pp. 305-335). 
Secondo Piero Gobetti Emma Gramatica è l'attrice che più «ha amato e seguito» Eleonora Duse (Gobetti, 1974, p. 33). Per lei come per Ruggeri e Musco Meldolesi ha coniato il termine di attori passatisti: la parola non ha valenza dispregiativa, indica il conflitto affrontato da questi artisti che possedevano tutte le abilità degli attori precedenti ma non avevano più lo spazio per esprimerle, mentre dominava le scene l'effervescente e certamente brava Dina Galli. Loro, come quelli affermatisi poco dopo, negli anni Venti: Maria Melato, Petrolini, Viviani e Benassi, più consapevoli forse della necessità di tutelare margini di indipendenza in una situazione che sempre più chiedeva attori e attrici funzionali a realizzare prodotti uniformati (Meldolesi, 2008, pp. 17-36). Le punte sono Irma ed Emma Gramatica, Maria Melato, ma le altre?

Dalle interviste che ho fatto a venticinque attrici nate fra il $1887 \mathrm{e}$ il 1923, di cui dieci erano figlie d'arte e quindici erano entrate in arte fra il 1904 e il 1933, risulta che ci fu consapevolezza dei cambiamenti epocali in corso e dei costi particolari pagati dalle donne (Mariani, 1987). $\mathrm{Fu}$ un processo di dissolvenza del vecchio teatro nel nuovo, in cui la tradizione mostrò i suoi limiti mentre la novità registica stentava tra condizionamenti e opacità. Eppure queste testimoni, benché colpite da una traumatica riorganizzazione professionale che produsse confusione e spaesamento, riuscirono a mantenere un buon rapporto con il pubblico, un notevole prestigio professionale e la consapevolezza della loro identità d'artiste. Certo dai loro racconti non emerge la realtà teatrale del primo Novecento, ma il suo ricordo: un mondo evocato, che attraverso la memoria sembra recuperare la sua ricchezza, come non è possibile attraverso i documenti d'epoca. Per questo è un mondo reale, da ripensare non solo nella superficie degli spettacoli e alla luce delle grandi perdite che ci furono, ma nella profondità dei suoi flussi di lunga durata e nella complessità di ciò che fu realmente e di ciò che avrebbe voluto essere. In questo senso possiamo ridimensionare il pessimismo di Schino a questo proposito per riaffermare la natura carsica della storia teatrale.

Meldolesi ha continuato a cercare di riempire la catena dell'attore-artista dopo Eleonora Duse e, occupandosi di Leo de Berardinis, ne ha tratto una conclusione sorprendente. Alla fine del ventesimo secolo, dopo i sommovimenti provocati dal Nuovo teatro, scrive che l'attore ar- 
tista è «la più significativa specificità italiana»: «L'attore artista italiano, che fa drammaturgia, forma nuovi attori, scopre spazi teatrali inopinati e crea altro pubblico, senza mai perdere di vista il suo carico di complessità sociale e culturale, è al centro del nostro sistema teatrale, anche quando agisce nell'emarginazione» (1994, pp. 9-10). È un'affermazione da approfondire, non foss'altro per la sicurezza con cui viene pronunciata da uno dei massimi studiosi dell'attore. Seguendone la suggestione, mi balza in mente un nome che può chiudere il secolo richiamando la prima attrice artista: Valentina Cortese (1923-2019). Un'attrice che ha dato molto anche al cinema, con un'aura divistica divenuta rara nel teatro, una straordinaria interprete di Ilse nei Giganti della montagna di Strehler, il personaggio che più di ogni altro incarna il mito dell'attriceartista ed evoca la Duse: «Il teatro si fa perché noi attori esistiamo, perché i poeti del teatro esistono solo per dire delle cose ad altri, alla gente. Ogni attore di teatro deve fare uno scarto, e creare un filtro poetico che sottragga al pubblico un po' del suo quotidiano» (Cortese, 2012, p. 183).

\section{RI-CREARE LA REALTÀ NEL TEATRO DI REGIA: RINA MORELLI, TITINA, LA MAGNANI}

Il decennio successivo alla Liberazione presenta un panorama contraddittorio: da un lato si afferma la generazione dei registi, fra cui i geniali Visconti e Strehler, mentre l'attore semplifica il suo linguaggio stimolato anche dal cinema, e, dall'altro, si diffonde uno stile livellato che vuole un attore buono per tutti gli usi, dallo stile pensoso, elegante, fintamente sincero. Questi caratteri stereotipati hanno aggravato le diffidenze, poi esplose, nei confronti dell'attore che interpreta un testo sotto la direzione del regista, come se questa condizione precludesse la creatività e l'autonomia. Stereotipi anche questi, su cui ha fatto chiarezza Gerardo Guerrieri (1975). Ci sono molti modi di essere attori - per genere, per repertorio e stile, per ruoli o emplois, per epoche e tradizioni teatrali ma la mediazione è un aspetto essenziale: l'attore media tra sé e il personaggio, tra l'autore e il pubblico. Si tratta di un esercizio non neutro né inerte, non necessariamente opposto alla creatività, essendo agito da un soggetto operante per metamorfosi continue di sé e del personaggio; 
anzi, proprio questa posizione porta l'attore a coltivare la sua differenza, isolandosi in casta, senza mai tuttavia poter prescindere dagli spettatori. L'attore nuovo nel tempo della regia è quello del "teatro della realtà", che assume l'interpretazione in quanto capacità critica e metamorfica, ridefinisce il proprio lavoro in relazione con il regista e tenta una sintesi delle qualità dell'interprete, fedele al testo, e del performer, che non si limita al livello verbale e tende al superamento dei limiti espressivi e rappresentativi del teatro di prosa.

Isabella Riva sostiene che Lilla Brignone e Rina Morelli impersonano «l'ultima generazione delle brave»: una generazione che ancora custodisce i valori delle famiglie d'arte ma si muove senza sentimentalismi nel territorio nuovo del teatro di regia (Mariani, 1987) ${ }^{14}$. L'indicazione è preziosa, perché non è facile individuare nel manipolo delle attrici significative del secondo dopoguerra quelle con funzioni di guida; trova inoltre conferma nelle recensioni dell'epoca e con forza particolare in quelle di De Monticelli (1976) che indica un primato di Rina Morelli (1908-1976). Scrivendo il suo necrologio, il critico usa parole inequivocabili: è «una delle più grandi attrici di questo secolo - e certo la più interiore, la più segreta e intensa», diversa dalle grandi «attriciflauto» e dalle «donne terribili», come Maria Melato.

Fu lei, insieme a Paolo Stoppa, a chiedere nel 1945 a Luchino Visconti di dirigerli in Antigone di Anouilh e in A porte chiuse di Sartre, avviando un lungo sodalizio. In un'epoca in cui gli attori erano insicuri e timorosi di perdere le doti del mestiere, Visconti si distingueva perché li seguiva uno per uno, li aiutava a spogliarsi di se stessi per cercare i sentimenti del personaggio, li circondava di cose vere e li stimolava a furia di dettagli, inventava tutto sul palcoscenico dove i personaggi vivevano: «Che cosa ci ha insegnato? Regole semplici, il lavoro, 1'odio per la genialità, la minuziosità», sintetizza Guerrieri (Geraci, 2006, pp. 69-73). Qualità possedute in sommo grado da Rina Morelli: antidiva che al solido mestiere affiancava la ricerca di un realismo antideclamatorio e umanissimo. In Zoo di vetro - recensisce Guerrieri - l'attrice riassumeva «la commedia in quegli sguardi accorati, passetti silenziosi,

14 Ho intervistato l'anziana attrice più volte nel 1984 a Bologna. 
diafane immobilità piene di luce» (1950, p. 89). Un'altra retorica, si può obiettare, ma che apriva la strada a una recitazione in levare, rendeva magnetica la leggerezza, emozionava il pubblico.

Su questo terreno di ricerca - un teatro vicino alla vita comune non possiamo non rivolgerci al teatro dialettale maggiore, dove si forma e opera principalmente un'attrice che considero fra le grandi del Novecento, Titina De Filippo (1898-1963). «Quando entra in campo, come i grandi contrattacchi fa subito goal» (Ibidem, p. 165), al pari dei fratelli è abilissima a legare comico e drammatico e, se Peppino incarna principalmente l'anima comica e Eduardo quella drammatica, Titina è grande sia nel varietà che nella prosa: in quel teatro «da ridere» dove serpeggiano «veleni anche più acuti di quelli che amareggiano il teatro di Cechov» (Savinio, 1938, p. 336). La sua provenienza dal teatro dialettale, se così si può chiamare il teatro napoletano, costituisce il nocciolo duro della sua identità artistica, questo significa legame con la realtà, ricerca di spontaneità, tensione a una precisione accalorata: Titina svela l'anima poetica del vituperato naturalismo, l'energia sottile che l'arte può succhiare dalla realtà. Attrice napoletana e universale al tempo stesso, con un mestiere di ferro ma senza mai perdere il contatto con la strada, portatrice di umanità, della sua personale umanità, in estrema riservatezza e tutela della propria vita privata, un'antidiva popolarissima. La testimonianza più bella a questo proposito l'ha lasciata Alberto Savinio:

Questa attrice che passeggia sulla scena con la compostezza di un possidente che passeggia nel proprio orto, e stacca le foglioline morte, svelle le gramigne, raddrizza i ramicelli, avvolge al sostegno le spire dei fagioli, c'ispira la confidenza e assieme l'indefinibile imbarazzo delle persone che ci hanno visto nascere, che ci hanno tenuti in braccio quando eravamo in fasce e le nostre labbra odoravano di latte. [...] Quale sorpresa però trovare attori che di là dalla ribalta serbano la densità, la gravità, la dignità della creatura umana! Questa la qualità maggiore dei De Filippo: Edoardo è "un uomo". Peppino è "un uomo". Titina è "una donna". I quali continuano sulla scena la fatalità della vita, e che senza raschiature né ripulimenti possiamo ritrovare fuori della scena. (Ibidem, pp. 263-265)

La sua maggiore interpretazione è Filumena Marturano nel testo omonimo di Eduardo. Ci sono pochi personaggi femminili altrettanto 
potenti nel teatro del 900. In tono minore, più sommesso, come si addice alla drammaturgia dei decenni centrali del secolo, Filumena è una padrona di casa e di bottega energica come la locandiera di Goldoni; è duramente provata dalla vita e dalla guerra, come la Madre coraggio brechtiana; ricorda Antigone perché segue una legge sua, che non coincide con quella dello stato; come Nora di Casa di bambola, scatena dibattiti a non finire. Il processo di lavoro per arrivare a questo trionfo non è lineare. Eduardo pare abbia paura di quello che ha scritto e «davanti alla forza di Filumena», retrocede, cerca dei compromessi, chiede a Titina di «reprimersi» (Carloni, 1984, pp. 115-146) ${ }^{15}$. Lei, pressata dalle indicazioni registiche del fratello, si blocca, non riesce a far suo il personaggio in profondità. La sera della prima al Politeama di Napoli Titina recita «come un automa sotto lo sguardo» di Eduardo e riscuote un successo tiepido. Consapevole dei suoi limiti interpretativi, timidamente chiede il parere del fratello: è stata una «schifezza», dice lui senza mezzi termini. Il giorno dell'ultima replica napoletana Eduardo convoca gli attori per una prova, ancora cerca di correggerla, non gli va bene niente. Titina si sente stanca e umiliata, Filumena rimane «rintanata» dentro di lei. Alla fine, messa alle strette l'attrice reagisce, chiede di recitare come sente, non vuole essere il «grammofono»del regista. Quella sera è un trionfo. «Simile a un proiettile mi ero lanciata e non mi fermava più nessuno. (...) Eccolo il mio personaggio. Lo avevo ghermito, palpitava nelle mie mani come una farfalla, e lo stringevo, lo stringevo dicendogli con gioia: finalmente, grida, urla, piangi... Ecco, così ti volevo: violenta, fredda, calma, tragica, comica» (Ibidem).

Ricordiamo a questo punto la formazione di Titina: la scena dialettale l'ha portata a costruire una lingua legata alla realtà, a un mondo di sentimenti e di cose oltre che di parole e di gesti; il varietà l'ha forgiata nel rapporto diretto, senza sconti, con il pubblico; il cinema ha valorizzato la naturalezza con cui abita il palcoscenico, da figlia d'arte. Da qui viene la sua capacità di mettere in scena la vita e di essere vista come persona prima che come attrice. Si delinea così una linea

15 Augusto Carloni, figlio di Titina, si basa sul diario che lei non ha voluto pubblicare. 
recitativa diversa da quella dell'attore artista, che può contare sulla bravura di Titina ed è penalizzata dalla sua ritrosia, ma può avvalersi del protagonismo indiscutibile di un'attrice non considerata dai nostri novecentisti, attratti in maggioranza dalle grandi e ben studiate teorie teatrali più che dalle sperimentazioni attoriche. Si tratta di Anna Magnani (1908-1973), un'attrice da riconquistare alla storia del teatro, che condivide con Titina alcuni tratti salienti: l'esperienza nel varietà e una base territoriale rivendicata, nella romanità questa volta, che si esprime poeticamente soprattutto nel canto.

Nel 1945, quando Roma città aperta di Rossellini la impone e la sua morte sullo schermo diventa la scena inaugurale del nuovo cinema italiano e del neorealismo, la Magnani (come si fa chiamare) ha alle spalle una solida carriera teatrale. Ha frequentato la Reale Scuola di Recitazione Eleonora Duse nel 1927-1928; ha recitato in compagnia Niccodemi con Vera Vergani (un tirocinio come generica poi rivendicato) e nella compagnia Antonio Gandusio-Luigi Almirante; è stata la protagonista di Anna Christie di O’Neill nel Teatro delle Arti di Bragaglia; è diventata una regina delle riviste di Michele Galdieri al fianco di Totò.

Parallelamente lavora nel cinema ma anche quando questa diventa la sua attività principale viene riconosciuta la sua sapienza teatrale. Lo sottolinea Jean Renoir, che la dirige ne La carrozza d'oro (1951): «Le si può attribuire la qualifica di comédienne, e io sono felice che essa voglia simboleggiare nel mio film tutti i comédiens del mondo (...). Anna è la quintessenza dell'Italia, è la personificazione assoluta del teatro, quello vero con gli scenari di cartapesta, le lampade fumanti, gli orpelli degli ori scoloriti!» (Hochkofler, 2013, pp. 156-158). E non solo di quel teatro, come mostra una delle sue prove maggiori, ne La voce umana di Cocteau, dove la presenza scenica diventa cinema, si esalta nel cinema, come sottolinea Rossellini, regista del film:

Meglio di ogni altro soggetto, Una voce umana mi offriva l'occasione di usare la macchina da presa come un microscopio, tanto più che il fenomeno da scrutare si chiamava Anna Magnani. Solo il romanzo, la poesia e il cinema permettono di scrutare nei personaggi per scoprire le loro reazioni e le cause che li fanno agire. L'esperimento spinto fino in fondo mi servì in tutti gli altri film, perché in un momento qualsiasi della realizzazione io sento 
il bisogno di lasciar da parte la sceneggiatura per seguire il personaggio nei suoi più segreti pensieri, quelli di cui forse non ha neppure coscienza. (Ibidem, pp. 92-93)

Del valore di questa interpretazione si rese ben conto Silvio d'Amico, mostrando una capacità rara di guardare gli attori, anche smentendo se stesso:

[...] E a questo punto perché non confessare, anche da parte nostra, che il nostro ideale di attrice moderna sarebbe l'opposto di ciò che offre, per lo più, Anna Magnani? Quando si sia ricordato che i nostri personali entusiasmi di spettatori sono stati soprattutto per l'ultima Duse, e per Ludmilla Pitoëff - vale a dire, per due attrici divenute tutto spirito, capaci di evasione e liberazione e purificazione sino all'estasi - ci vuol poco a capire che il verismo della solita Magnani scarmigliata, e specialmente della Magnani nella Voce umana, è, in partenza, alle mille miglia dalle nostre inveterate predilezioni.

Ma qui appunto sta il miracolo di questa creatrice di portenti: che proprio attraverso quello spoglio e violento verismo essa attinge a suo modo un Dilà, un mistero e, ridiciamo la parola grossa, una poesia. Nella Voce umana, che, come tutti sanno, rappresenta l'ultima telefonata d'una povera donna disfatta, all'amante che l'ha piantata per sposarsi con un'altra - oh, senza più nessun bisogno d'insistere sul romanesco - si getta nella passione così a capofitto, squassando carne e anima, con una disperazione così fonda, da far tremare l'atmosfera. E da quei suoi schianti prorompe il più accorato, il più vasto pianto umano: il che non si chiama Nannarella, si chiama arte. (2005, vol. III, pp. 653-656)

Da questo livello - in cui la ricerca di un teatro altro per un pubblico non di nicchia fa incontrare Eleonora Duse con Totò e pure con la Magnani - possiamo partire per un approfondimento del secolo, perché «la regia non basta a qualificare il Novecento teatrale, neppure se estesa all'attore-regista». Varie attrici hanno pure creato la loro «arte superatrice, dando vita a altrettante storie altre», universalizzando mirabilmente «nella forma la loro condizione costretta». Così leggiamo in un appunto inedito di Claudio Meldolesi che, dopo Eleonora Duse e Sanjukta Panigraj, la danzatrice Odissi appena morta, citava: «Helene Weigel, Stella Adler, Titina De Filippo, Maria Casarès, Iben Nagel 
Rasmussen o Glenda Jackson o Irene Papas ${ }^{16}$. Pensava soprattutto alla figura dell'attrice-artista, ma accanto a questa possiamo registrare una fisionomia diversa: di un'attrice che, superate le rotture traumatiche degli anni Trenta, accetta la direzione del regista senza rinunciare ai suoi bisogni d'arte e d'indipendenza; che andando oltre le contrapposizioni (o gli adeguamenti di superficie) fra teatro e cinema, si avvale di esperienze teatrali e cinematografiche insieme; che è interessata al presente ed è consapevole della difficoltà di tradurlo scenicamente; che si riallaccia alla tradizione preregistica più avanzata e aperta, forse inconsciamente memore di un Ottocento in cui le attrici hanno potuto dirigere la compagnia e gestire il loro repertorio. Titina però non ha avuto il coraggio di fare la capocomica e Anna Magnani ha dovuto lottare duramente per non trasformarsi in una maschera, per avere personaggi «nei quali poter credere, a cui il pubblico possa credere. Personaggi ben costruiti, senza squilibri di artifici e fasullaggini. Veri. Veri vuol dire personaggi presi dalla vita: nella vita troppi ce ne sono di personaggi cui ispirarsi!» (Hochkofler, 2013, p. 100).

Questa ricerca di verità è anzitutto del cinema: «La nuova professionalità dell'attore neorealista si configura essenzialmente come necessità di adesione al proprio personaggio: sul piano fisiognomico, prima che comportamentale e interpretativo», soprattutto «a partire dalla possibilità che un volto e un corpo siano già in grado di essere il personaggio, di raccontare una storia» (Grignaffini, 2002, p. 272). Questa pratica della «naturalizzazione della recitazione» (Ibidem) affonda in una tradizione teatrale consolidata ma nel cinema si realizza con la supremazia del regista, a differenza che nel teatro. Converrà dunque tornare all'esperienza di Anna Magnani con Totò, per ricordare l'identità di attore indipendente di quest'ultimo: un attore che si dà pienamente sulla scena e recita con energia superiore alle necessità rappresentative, che è per definizione unico pur non recitando da solo, che anche negli spettacoli firmati da un regista crea uno spettacolo nello spettacolo, un film nel film, tutti suoi, di cui potrebbe firmare anche la drammaturgia e la regia.

${ }^{16}$ Il foglio, presumibilmente del 1997, è conservato presso l'Archivio privato di Claudio Meldolesi a Bologna. 
Totò definì la Magnani «donna di cappa e spada», una donna cioè «la cui forza in scena era tale da costringerlo a rilanciare i suoi consueti attacchi di seduzione», anche lei amava improvvisare o - come diceva «essere una sera in un modo e una sera in un altro». Totò «recitava come una maschera rimanendo sempre se stesso, ma quel se stesso d'infinite possibilità in cui consiste l'attore creatore» ha scritto Cavicchioli (Meldolesi, 2013, p. 30), ugualmente Anna Magnani a detta di D'Amico, «prodigiosa nella mimica, mobilissima, d'un volto assai più che bello; impagabile nell'invettiva, nel sarcasmo, negli strazi del singulto; maschera, insomma, sua». Per questo come Totò ha bisogno del suo doppio sociale, il principe de Curtis, la Magnani si serve di Nannarella. Ma se «Zago era Venezia, Viviani era Napoli, Grasso e Musco erano Catania; e quattro quinti della loro recitazione (se proprio si abbia da chiamarla cosi) erano bell'e fatti con la semplice presenza fisica delle loro persone», nella Magnani tutto questo è ri-creazione: «essa recita il tipo che si è creata; e così bene, da dar l'illusione della verità» (D'Amico, 2005, pp. 653-655), come i nostri comici dell'Arte.

\section{ALCUNE FIGURE NEL PANORAMA AFFOLLATO E SFUGGENTE DI FINE SECOLO}

Questo paragrafo ha un carattere quasi testimoniale, riferendosi ad anni non sufficientemente storicizzati dal punto di vista del genere. Riguarda il periodo successivo all'emersione della seconda avanguardia storica internazionale e allo scoppio di ampi sommovimenti politici, fra lo storico Convegno di Ivrea del 1967, che dà visibilità al Nuovo che emerge e alle sue esigenze di organizzazione, e la fine degli anni Ottanta, quando ha preso piede il Nuovo Teatro, in tale polemica con la scena esistente che molte non si riconoscono nella parola attrice e nel mestiere cui si riferisce. L'artista di riferimento è Perla Peragallo (1943-2007), che si impone ne La faticosa messinscena dell'Amleto di Shakespeare, firmata insieme a Leo de Berardinis e presentata a Ivrea appunto: un Amleto sovvertitore, che distrugge il testo per restituirlo potenziato dalle invenzioni musicali e vocali, visive e cinematografiche, e mostra la potenza magnetica di Perla, confermata negli spettacoli successivi, 
finché nel 1981 lei si ritira dalle scene e lui inizia la sua "terza vita" artistica a Bologna. Un rapporto di coppia dagli equilibri particolari, paritetici nelle diversità, più di quanto traspaia in altre celebri coppie: Carmelo Bene-Lydia Mancinelli, Carlo Quartucci-Carla Tatò, Giancarlo Nanni-Manuela Kustermann, nonostante la bravura delle attrici e il loro ruolo indiscusso nel teatro d'avanguardia.

Volendo accatastare, in una scansione paratattica, in un unico lungo elenco, i vari tentativi di definizione dei ruoli di cui si farebbero carico Leo e Perla nel loro violento e dolente 'corpo a corpo', si può dire che il primo è leader, regista in scena, conduttore, guida, spalla, presentatore-attore, responsabile del controllo della situazione; la seconda è invece perno, centro vitale, punto di riferimento, martellata, basso continuo, batteria, bestia da palcoscenico, unico attore in scena [...]. "Lei appunto non "fa' teatro ma 'è' teatro" ${ }^{17}$. (Vassalli, 2018, pp. 62-63)

Più aspetti della recitazione di Perla sono di svolta: non interpreta entità psicologicamente definite, ma parte dal suo sentimento tragico della vita e dalla sua rabbia, investe tutta se stessa nello scavo di "stati di coscienza", secondo il termine poi usato da Leo. Inoltre, punta sulla voce e sulla costruzione di una partitura musicale: dice di possedere 3600 voci e le usa con modalità inedite, con la sapienza di una che tra le note è cresciuta. Sicché le attrici che si richiamano a Carmelo Bene e alla phoné farebbero bene ad allacciarsi a Perla, anche se ha lasciato poche testimonianze pubbliche.

Nell'Archivio Leo de Berardinis del Dipartimento delle Arti dell'Università di Bologna, sono conservati sei quaderni suoi, relativi ad altrettanti spettacoli presentati fra il 1972 e il 1976. Sono esempi di «scrittura scenica come partitura musicale», per usare parole di Leo, che restituiscono struttura dello spettacolo, battute verbali, suoni, luci, spazi, movimenti, oggetti: una drammaturgia composita, in larga misura nuova per il pubblico del tempo, «concreta e al tempo stesso poetica e visionaria», illustrata anche con disegni, e che prevede momenti di improvvisazione (Biasin, 2019). Ma ciò che «teatralmente faceva esplodere e lo ha fatto

${ }^{17}$ La prima monografia su Perla si deve a Maximilian La Monica. 
nel bene e nel male, era proprio che in scena c'era Perla Peragallo» (de Berardinis, 1987): qualità confermata dalle poche immagini che restano e dalle parole di critici d'eccezione come Ripellino o Guerrieri.

Perla prosegue e rinnova la linea dusiana per il suo bisogno di purezza e la sua insofferenza dei limiti, conferendo centralità alla voce e pienezza alla presenza anche silente, però eccedendo in passionalità come in rigore. Dopo di lei sono attrici artiste di un Teatro di voce Ermanna Montanari ed Elena Bucci, per filiazione diretta quest'ultima, avendo ricevuto da Perla i citati quaderni ed essendo stata un'attrice di de Berardinis, mentre Ermanna Montanari con Chiara Guidi e Mariangela Gualtieri dà splendore alla "Romagna felix" degli anni Ottanta. Tutte le migliori attrici del Nuovo Teatro sono sperimentatrici di linguaggi, per loro potremmo usare le parole proposte per Adelaide Ristori da Simoncini nella sua relazione al convegno: manifestano un senso «di onnipotenza scenica», occupano l'intero palcoscenico prendendo in pugno la platea, anche quando nella vita sembrano "attrici di". È così per la mitica Marion d'Amburgo, troppo presto appartatasi dai Magazzini, portatrice di sostanza umana nell'avanguardia più spericolata, come per le gemelle Pasello: materializzazione di un doppio che esalta il valore dell'ambiguità scenica, drammaticamente tornato a unità ora che Silvia non ha più accanto Luisa (1957-2013). Le sorelle richiamano Grotowski e Thierry Salmon, in particolare Le Troiane dirette da quest'ultimo nel 1988 e animate da presenze femminili eccezionali: numerose attrici di varia nazionalità, tra cui Maria Grazia Mandruzzato e Renata Palminiello, e la dramaturg Renata Molinari, con le musiche e la direzione del coro di Giovanna Marini.

Da posizioni ugualmente impegnate nella ricerca alcune attrici napoletane rilanciano l'eredità di Titina anche per la loro capacità di essere efficaci e vere sulla scena come sullo schermo: da Isa Danieli - attrice di Eduardo e dello storico La Gatta Cenerentola di De Simone, vincitrice di un Ubu per la sua Filumena Marturano nel 2001 - a Licia Maglietta e Iaia Forte. A loro accosterei la torinese Laura Curino che, non a caso, negli anni Novanta si rimanifesterà nel contesto diverso del Teatro di narrazione, su tutt'altro piano rispetto alla sua conterranea Maria Luisa Abate. 
D'altro canto, molte riversano il loro impegno di ricerca nel lavoro di interpretazione, ad esempio Marisa Fabbri (1927-2003), attrice ronconiana, che si muove con sicurezza fra tradizione attorica e teatro di regia, fra mestiere e ricerca, fino a trasfigurare il ruolo di caratterista, cui sembrava destinata dal suo aspetto, per aderire alle arditezze di Luca Ronconi e nutrirsi intellettualmente dello strutturalismo più intransigente come del pensiero di Gramsci e Lukàcs (Longhi, 2010). Marisa Fabbri vince la difficile battaglia di sviluppare dal ceppo della tradizione un attore moderno, all'altezza delle sfide poste dalla regia e poi dal Nuovo Teatro, affermando il valore trasversale della ricerca e dunque l'unità del teatro nella diversità. Sarebbero molte le interpreti di questo tipo da citare per gli anni successivi: a cominciare dalle straordinarie Maria Paiato e Anna Bonaiuto per finire con le più giovani Manuela Mandracchia, Alvia Reale e Sandra Toffolatti, che abbiamo visto nel video presentato al nostro convegno ${ }^{18}$. Hanno dato vita a una compagnia autonoma e l'hanno chiamata Miti pretese, nome che sembra nascondere una questione vecchia di decenni: la ritrosia delle attrici anche più brave e autonome ad assumere posti di comando nella compagnia o nel gruppo.

È difficile anche la scalata alla regia, come mostra già la prima delle registe, l'inglese Edith Craig, penalizzata dal fatto che concepì questo ruolo in continuità con la tradizione e dette importanza alle pratiche più che alla formalizzazione scritta delle idee. In Italia, dopo Wanda Fabro, morta nel 1943 a 34 anni, con cinque regie al suo attivo, dobbiamo aspettare alcuni decenni per trovare un numero di registe importanti: André Ruth Shammah, Cristina Pezzoli, Monica Conti, Emma Dante, Elena Bucci, Serena Sinigaglia... dove Dante domina sui colleghi uomini. Molto più numerose le attrici che curano la regia dei propri assolo mentre fra le registe alcune recitano o hanno recitato, come Emma Dante, Elena Bucci, Lucia Calamaro... e Dante e Calamaro sono anzitutto drammaturghe (Mariani, 2016).

18 Il video, realizzato appositamente per il convegno di Cracovia su indicazione di Teresa Megale, consente di ricostruire la particolare drammaturgia di gruppo perseguita dalle tre artiste, nella convinzione che sia necessario un teatro fondato su una prospettiva di genere. Propone brevi stralci tratti dagli spettacoli Roma ore 11, Troiane, Festa di famiglia. 
È aumentato infatti il numero delle attrici che scrivono. Certo il fatto stesso che scriva una donna fa emergere nuove tematiche, nuovi personaggi, ma per tutte si pone il problema del linguaggio: trovare parole proprie in un vocabolario costruito dagli uomini, accettare l'impossibilità di dire e il silenzio, partire dal corpo come avviene sulla scena, coltivare le differenze, perché «il linguaggio umano è uno (...). Ma forse le segrete leggi del ritmo hanno un sesso», come osserva Sibilla Aleramo (1922, pp. 55-66), autrice di drammi non significativi quanto il resto della sua produzione ma ben consapevole della forza del teatro e delle attrici.

Anche se le rigidità connesse agli statuti dei generi letterari sembra che neghino alle drammaturghe le libertà che le attrici si prendono sulla scena, non mancano le figure di primo piano, da Dacia Maraini a Natalia Ginzburg, mentre l'affermarsi della "scrittura scenica" libera energie fresche, anche in virtù della priorità assunta dalla scena, come mostrano Dante e Calamaro. Un discorso a sé meriterebbero le attrici comiche anche autrici, a cominciare da Franca Valeri: lontane eredi di quella Mirandolina che Megale (2008) ha individuato come prototipo della comicità femminile per le servette e poi per le primedonne e come esempio di autonomia femminile nel teatro oltre che nella società in generale. Si afferma cioè via via una tendenza, che si manifesterà pienamente all'inizio del secolo successivo, ad essere donne di teatro globali e ad assumere i ruoli fluidamente.

Ma se finora abbiamo parlato soprattutto di quanto le attrici hanno dato alle donne, non possiamo non ricordare il debito sul versante opposto, nei confronti del pensiero femminista: che ha posto l'attenzione sul soggetto che enuncia, rifiutando le assimilazioni astratte ed essenzialistiche; che, a partire dall'individualità singolare di ogni essere umano, ha contrapposto all'astratta singolarità del discorso l'incarnazione storica del suo soggetto e ha immesso la soggettività femminile come misura della ricerca e la memoria del corpo fra i suoi presupposti (Luisa Passerini, Adriana Cavarero); ha proposto un soggetto nomade, che attraversa i saperi, evitando sia la complementarietà degli opposti che l'utopia dell'altrove (Rosi Braidotti); ha messo in evidenza il carattere performativo dei generi sessuali (Judith Butler). 


\section{BIBLIOGRAFIA}

Aleramo, S. (1922). Apologia dello spirito femminile. In Eadem, Andando e stando. Firenze: Bemporad.

Aliverti, M.I. (1992). Poesia fuggitiva sugli attori nell'età di Voltaire. Roma: Bulzoni.

Apollonio, M. (2003). Storia del teatro italiano. Dall'età barocca al Novecento, nuova edizione integrata. Milano: Rizzoli.

Attisani, A. (2015). L'arte e il sapere dell'attore. Idee e figure. Torino: Accademia university press.

Attisani, A. (2017). L'invenzione del teatro. Fenomenologia e attore della ricerca. Imola: Cue Press.

Biasin, S. (2019). I quaderni di Perla. Culture Teatrali, 28, 98-112.

Braidotti, R. (1995). Soggetto nomade. Femminismo e crisi della modernità. Roma: Donzelli.

Butler, J. (2013). Questioni di genere. Il femminismo e la sovversione dell'identità. Roma-Bari: Laterza.

Carloni, A. (1984). Titina De Filippo. Vita di una donna di teatro. Milano: Rusconi.

Cavarero, A. (1995). Corpo in figure. Filosofia e politica della corporeità. Milano: Feltrinelli.

Cavarero, A. ( 2003). A più voci. Filosofia dell'espressione vocale. Milano: Feltrinelli.

Cecconi, A. (Ed.). (2013). Il genere del pubblico: questioni per una storia della ricezione femminile nelle arti della scena. In L. Guidi \& M.R. Pellizzari (Eds.), Nuove frontiere per la Storia di genere (vol. 3, pp. 181-214). Salerno: Università di Salerno e Libreria universitaria.it.

Ciotti Cavalletto, G. (1978). Attrici e società nell'Ottocento italiano. Miti e condizionamenti. Milano: Mursia.

Cortese, V. (2012). Quanti sono i domani possibili. Milano: Mondadori.

D’Amico, S. (2005). Anna Magnani. In A. d'Amico \& L. Vito (Eds.). Cronache 1914/1955 (vol. 5, t. III, pp. 653-656). Palermo: Edizioni Novecento.

De Berardinis, L. (1987). Il teatro è l'attore. Ma l'attore dov'è? In A. Attisani (Eds.), Le forze in campo (pp. 55-59). Modena: Mucchi editore.

De Monticelli, R. (2019). Rina Morelli, una figlia d'arte con la paura del palcoscenico. In G. De Monticelli (Ed.). L'attore. Retrieved from www. cuepress.com. 
Duse, E., \& Boito, A. (1979). Lettere d'amore, a cura di R. Radice. Milano: Il Saggiatore.

Gandolfi, R. (2003). La prima regista. Edith Craig, fra rivoluzione della scena e cultura delle donne. Roma: Bulzoni.

Garboli, C. (1998). Un po' prima del piombo. Milano: Sansoni.

Geraci, S. (Ed.). (2006). Il teatro di Visconti. Scritti di Gerardo Guerrieri. Roma: Officina Edizioni.

Geraci, S. (2010). Destini e retrobotteghe. Teatro Italiano nel primo Ottocento. Roma: Bulzoni.

Gobetti, P. (1974). Scritti di critica teatrale. Torino: Einaudi.

Grignaffini, G. (2002). La scena madre, Scritti sul cinema. Bologna: Bononia University Press.

Guerrieri, G. (1975), Attore. In Enciclopedia dello spettacolo (vol. 1, pp. 1074 -1093). Roma: Unione Editoriale.

Guerrieri, G., (1946). Tre moschettieri a Zoo di vetro. In R. Brancati (Ed.). (2016). Presagi per un teatro nuovo (pp. 88-89). Irsina: Giuseppe Barile Editore.

Hochkofler, M. (2013). Anna Magnani. La biografia. Milano: Bompiani.

Longhi, C. (2010). Marisa Fabbri. Lungo viaggio attraverso il teatro di regia. Firenze: Le Lettere.

Mariani, L. (1987). Donne di teatro nel primo Novecento (Ph.D. Thesis). Bologna: Università di Bologna.

Mariani, L. (2005). L'attrice del cuore. Storia di Giacinta Pezzana attraverso le lettere. Firenze: Le Lettere.

Mariani, L. (2012). Ermanna Montanari. Fare-disfare-rifare nel teatro delle Albe. Corazzano: Titivillus.

Mariani, L. (2016). Registe di teatro in Italia. Ce n'est qu'un début... Culture Teatrali, 25, 108-123.

Mariani, L. (2017). L'Ottocento delle attrici. Acting Archives Review, 14. Retrieved from www.actingarchives.it.

Megale, T. (2008). Mirandolina e le sue interpreti. Roma: Bulzoni.

Meldolesi, C., \& Taviani, F. ( 1991). Teatro e spettacolo nel primo Ottocento. Roma-Bari: Laterza.

Meldolesi, C. (1994). L'attore artista. I quaderni di Santarcangelo, 1, 9-10.

Meldolesi, C. (1995). Piazza e teatro. L'attore nell'età delle invenzioni. In M. Vitale \& D. Scafoglio (Eds.), La piazza nella storia: eventi, liturgie, rappresentazioni (pp. 413-420). Napoli: Edizioni Scientifiche Italiane. 
Meldolesi, C. (1996). Questo strano teatro creato dagli attori artisti nel tempo della regia, che ha rigenerato l'avanguardia storica insieme al popolare. Teatro e Storia, 18, 9-23.

Meldolesi, C. (2008). Fondamenti del teatro italiano. La generazione dei registi. Roma: Bulzoni.

Meldolesi, C. (2013), Pensare l'attore, a cura di L. Mariani, M. Schino \& F. Taviani. Roma: Bulzoni.

Passerini, L. (1988), Storia e soggettività. Le fonti orali, la memoria. Firenze: La Nuova Italia.

Pedullà, G. (1994), Il teatro italiano nel tempo del fascismo. Bologna: il Mulino.

Rivière, J. (1991), Womanliness as a Masquerade. In A. Huhes (Eds.), The Inner World and Joan Rivière. Collected Papers: 1920-1958 (pp. 99-101). London-New York, Karnac Books.

Ristori, A. (1887). Ricordi e studi artistici. Torino-Napoli: L. Roux.

Savinio, A., (1938). Teatro da ridere. In A. Tinterri (Eds.). (1982). Palchetti romani (pp. 334-337). Milano: Adelphi.

Schino, M. (1997). L'acquisto del teatro. Documenti sul caso Cortese. In A. Tinterri (Ed.), La passione teatrale. Studi per Alessandro d'Amico (pp. 305-335). Roma: Bulzoni.

Schino, M., (2008). Il teatro di Eleonora Duse. Nuova edizione riveduta e ampliata. Roma: Bulzoni.

Schino, M. (2016). Racconti del Grande Attore. Tra la Rachel e la Duse. Imola: Cue Press.

Schino, M., Di Tizio, R., Legge, D., Marenzi, S., \& Scappa, A. (2017). Teatri nel fascismo. Sette storie utili. Teatro e Storia, 38, 59-384.

Simoncini, F. (2011). Eleonora Duse capocomica. Firenze: Le Lettere.

Thebaud, F. (Ed.). (1992). Storia delle donne. Il Novecento. Roma-Bari: Laterza.

Talli, V. (1927). La mia vita di teatro. Milano: Fratelli Treves.

Taviani, F., \& Schino, M. (1982). Il segreto della Commedia dell'Arte. La memoria delle compagnie italiane del XVI, XVII e XVIII secolo. Firenze: La casa Usher.

Taviani, F. (2002). Attore e Attrice. In Enciclopedia del Cinema (pp. 322-337). Roma: Treccani.

Valenti, C. (2015). Lineamenti di un non movimento. Indagine sulla contemporaneità e nuovi paradigmi del politico nel teatro del terzo millennio. Culture Teatrali, 24, 132-144. 
Vassalli, A. (2018). La tentazione del sud. Viaggio nel teatro di Leo e Perla da Roma a Marigliano. Corazzano: Titivillus.

Viziano, T. (2000). Il palcoscenico di Adelaide Ristori. Repertorio, scenario e costumi di una Compagnia Drammatica dell'Ottocento. Roma: Bulzoni.

Zacconi, E. (1946). Ricordi e battaglie. Milano: Garzanti.

Zancan, M. (1998). Il doppio itinerario della scrittura. La donna nella tradizione letteraria italiana. Torino: Einaudi.

Riassunto: Gli studi critici sul teatro dell' 800 e del 900, anche quelli fondativi, hanno il limite di non usare la categoria di gender: un paradosso in un ambito in cui il corpo è centrale. Le attrici infatti danno vita a storie diverse, che permettono di arricchire le ricostruzioni storico-critiche. Data l'ampiezza del tema, il saggio punta sull'individuazione di alcuni snodi fondamentali: il lascito ottocentesco, ricco sia dal punto di vista del linguaggio che da quello socio-culturale; il dopo-Duse, che registra da un lato la nascita della specificità italiana dell'attore-artista e, dall'altro, la crisi del capocomicato femminile; la nascita della regia italiana nel secondo dopoguerra e la reazione di alcune attrici che puntano sulla ri-creazione della realtà, come Anna Magnani, che va riconquistata alla storia del teatro; la rottura operata dal Nuovo teatro e la messa in discussione sin della parola attrice, con sconfinamenti nei campi della regia e della drammaturgia.

Parole chiave: Ottocento, Novecento, teatro, attrice, storia delle donne 